\title{
Novel cadmium responsive microRNAs in Daphnia pulex
}

Shuai Chen ${ }^{1}$, Garrett J. McKinney ${ }^{2}$, Krista M. Nichols ${ }^{1,3}$, John K. Colbourne ${ }^{4}$ and Maria S. Sepúlveda**

${ }^{1}$ Department of Forestry and Natural Resources, Purdue University, West Lafayette, Indiana, USA

${ }^{2}$ School of Aquatic and Fishery Sciences, University of Washington, Seattle, Washington State, USA

${ }^{3}$ Conservation Biology Division, Northwest Fisheries Science Center, National Marine Fisheries Service, National Ocean and Atmospheric Administration, Seattle, Washington State, USA

${ }^{4}$ Environmental Genomics Group, School of Biosciences, University of Birmingham, Edgbaston, Birmingham, UK

${ }^{*}$ Corresponding Author: Maria S. Sepúlveda

Address: FORS Room 103

195 Marsteller St

West Lafayette, IN 47907-2033

Phone: 765.496 .3428

Fax: 765.496.2422

Email: mssepulv@purdue.edu

\section{SUPPORTING INFORMATION}

Summary

Number of pages: 21

Number of Tables: 6

Number of Figures: 3 

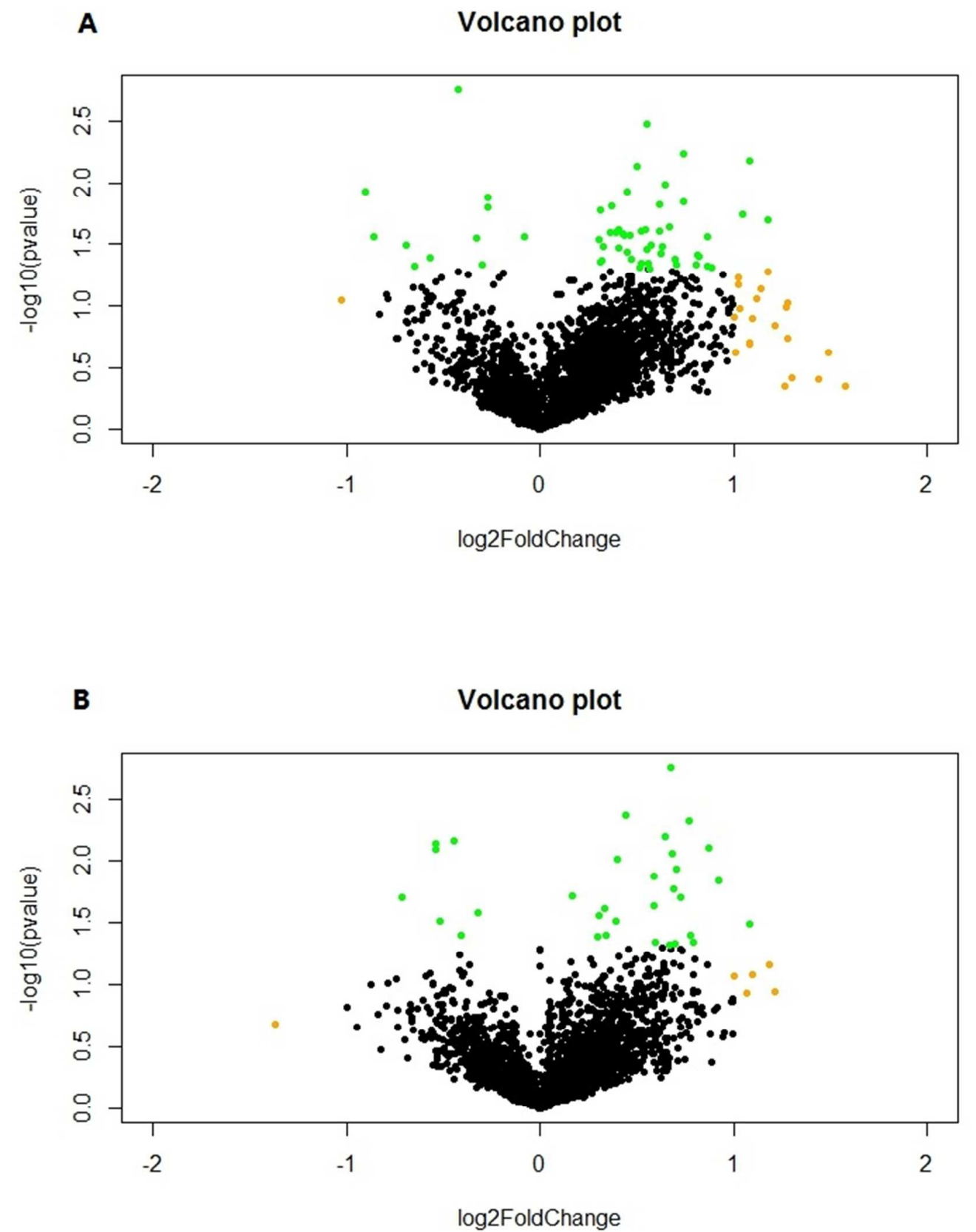

Figure S1. Volcano plots showing miRNA expression changes in Cd-low $(A)$ and Cd-high (B) groups compared with controls. Only miRNAs with $\log _{2} A E>2$ are shown. Orange dots represent miRNAs with $\log _{2} \mathrm{FC}>1$. Green dots represent miRNAs with $p$-value $<0.05$. $X$-axis is the $\log _{2}$ fold change in the Cd-low/Cd-high group compared to controls and Y-axis is the $-\log _{10}$ of $p$ values. 


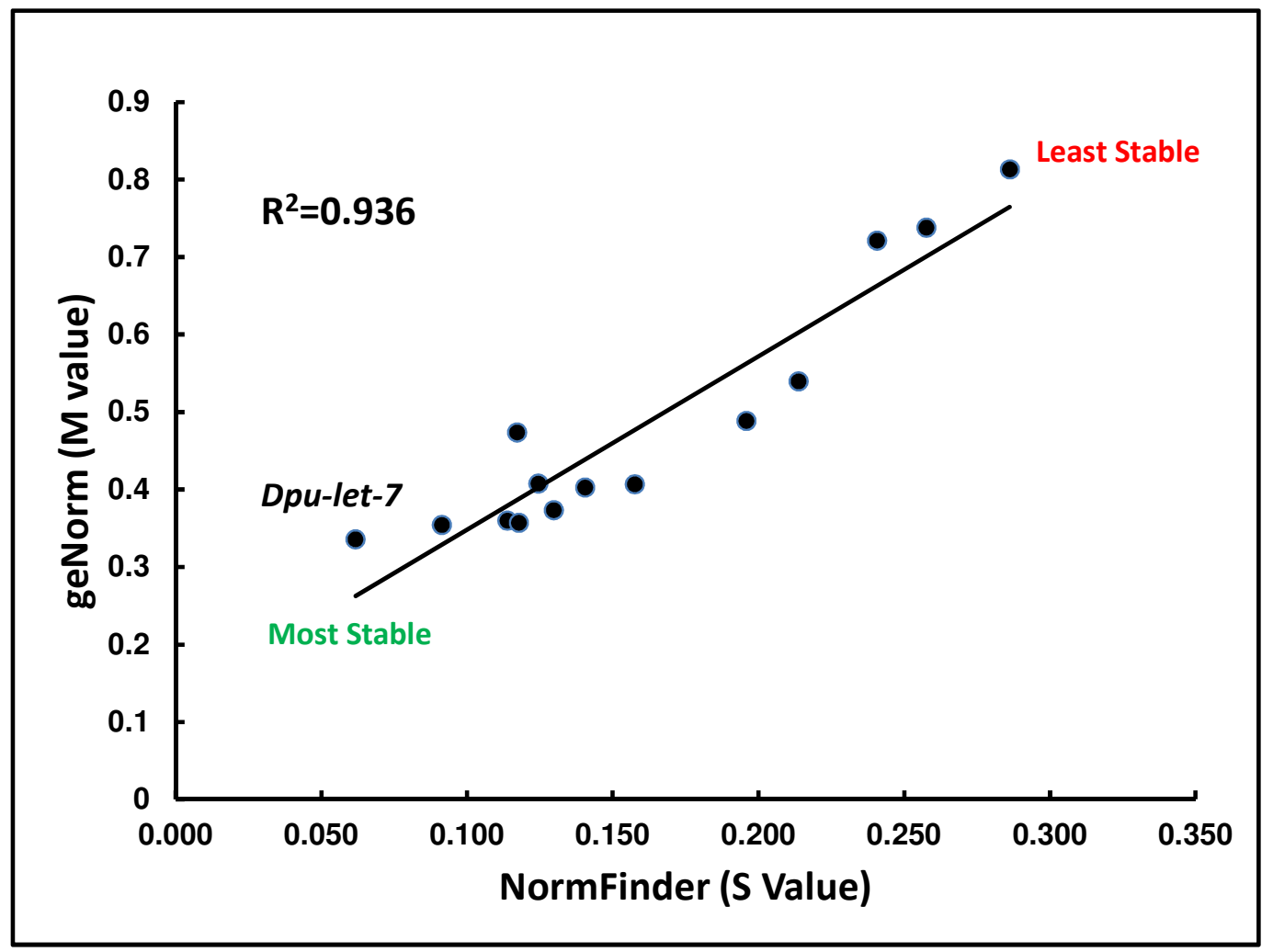

Figure S2. Correlation of $M$ value obtained using geNorm and $S$ value using NormFinder for 17 candidate reference genes. Dpu-let-7 was selected as the most stable expressed reference gene under our test conditions. 


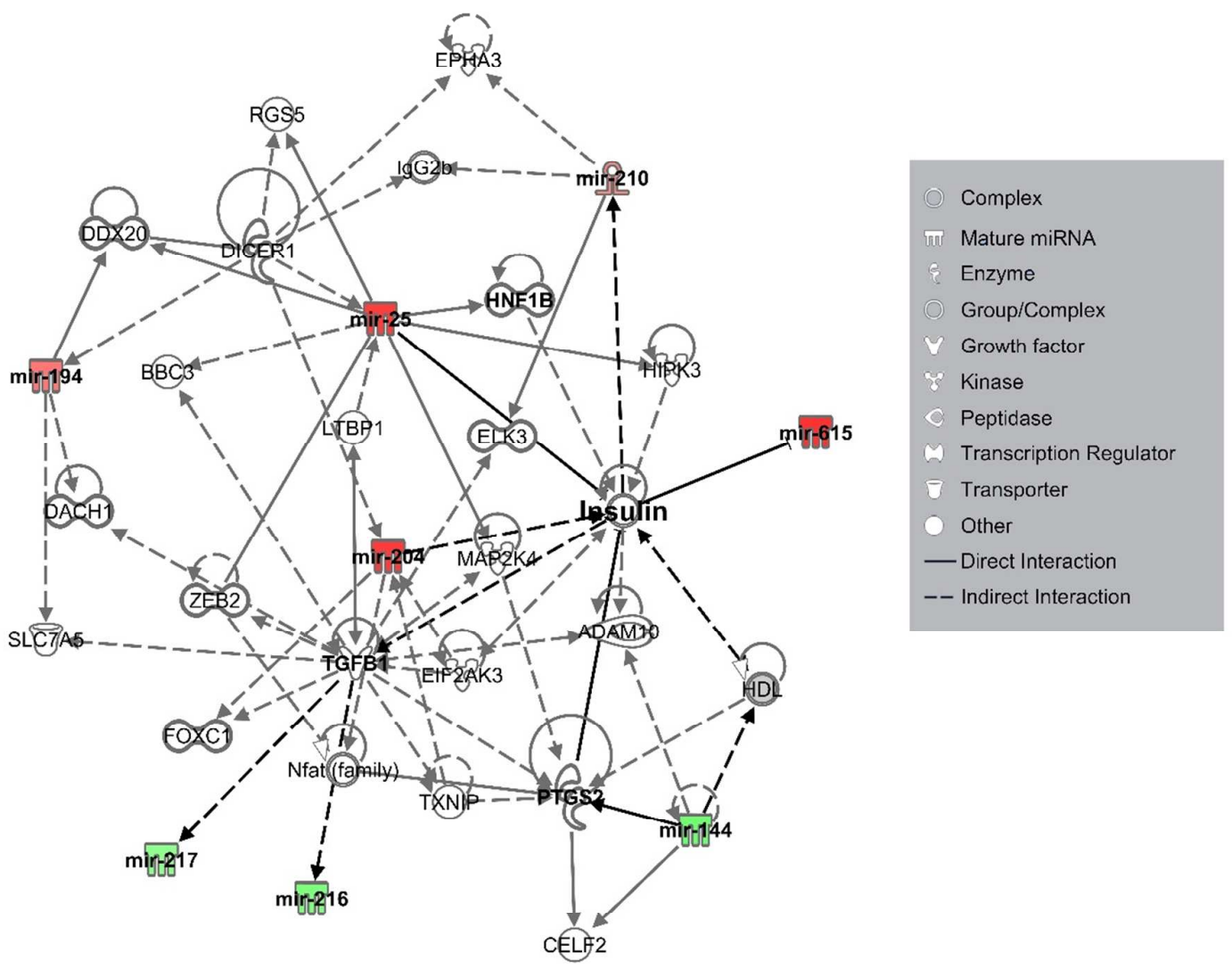

Figure S3. IPA analysis of significantly differentially expressed miRNAs revealed an insulin-network that is related with "organismal injury and abnormalities" (see Supplemental Table 4 for detailed gene symbols). Color intensity represents the degree of up (red) or down (green) regulation of miRNAs. 
Table S1. Predicted $D$. pulex miRNAs that were not covered by microarray.

\begin{tabular}{|c|c|c|}
\hline miRNA Name & Genome Loci & miRNA sequence \\
\hline dpu-mir-125 & scaffold_71:445406-445427 & UCACAAGUUAGGGUCUCAGGGA \\
\hline dpu-mir-1277a & scaffold_32:794746-794767 & AUAUAUAUAUAUAUAUAUGUAC \\
\hline dpu-mir-1277b & scaffold_100:300960-300981 & CUUUAUAUAUAUAUAUAUGUAC \\
\hline dpu-mir-1322 & scaffold_42:141666-141687 & ACAGCAACAACAGCAGCAGCAA \\
\hline dpu-mir-15 & scaffold_28:901796-901817 & CAGCAGCAGCACACAAAACUAU \\
\hline dpu-mir-1502 & scaffold_5030:534-552 & AGACUUUUCAGGUAGUUCG \\
\hline dpu-mir-1587a & scaffold_46:689049-689070 & GGGCUGGGCUGGGCUGGGCUGG \\
\hline dpu-mir-1587b & scaffold_200:123544-123565 & GGGCUGGGCUGGGCUGGGCUGG \\
\hline dpu-mir-1814 & scaffold_40:1045656-1045677 & GGUUUUUUGGUUUUGUUUCCUU \\
\hline dpu-mir-184 & scaffold_24:317115-317138 & ACUGGACGGAGAACUGAUAAGGGC \\
\hline dpu-mir-190 & scaffold_3:1681569-1681594 & AGAUAUGUUUGAUAUUCUUGGUUGUU \\
\hline dpu-mir-210-3p & scaffold_51:480364-480342 & CUUGUGCGUGUGACAGCGGCUAU \\
\hline dpu-mir-210-5p & scaffold_51:480344-480365 & AGCCGCUGUCACACGCACAAGA \\
\hline dpu-mir-2325 & scaffold_41:201387-201408 & GUUGUUUUUUUUCUUUUUUUUA \\
\hline dpu-mir-279 & scaffold_63:523674-523653 & UGACUAGAUCCACACUCAUCCA \\
\hline dpu-mir-282 & scaffold_57:216687-216666 & UAGCCUCUCCUAGGCUUUGUCU \\
\hline dpu-mir-2a & scaffold_80:240622-240644 & UAUCACAGCCAGCUUUGACGAGC \\
\hline dpu-mir-2b & scaffold_80:241083-241106 & UAUCACAGCCAGCUUUGAUGAGCG \\
\hline dpu-mir-307-3p & scaffold_47:641122-641102 & UCACAACCUCCUUGAGUGAGU \\
\hline dpu-mir-307-5p & scaffold_47:641084-641103 & UCACAACCUCCUUGAGUGAG \\
\hline dpu-mir-309 & scaffold_24:361445-361466 & AGUCACUGGGUAAAGUUUGUCC \\
\hline dpu-mir-313 & scaffold_32:710981-711002 & GAAUGUUGUGAAUAGUGUAAUA \\
\hline dpu-mir-341 & scaffold_53:695412-695431 & UCGGUCGAUCGGUCGGUGGU \\
\hline dpu-mir-4507 & scaffold_200:122781-122802 & CUGGGUUGGGCUGGGCUGAAUA \\
\hline dpu-mir-466a & scaffold_9:248987-249008 & AGAGACACACACACACACAAGU \\
\hline dpu-mir-466b & scaffold_19:1276861-1276882 & GUGUGUGUGUGUGAGUGUGUAC \\
\hline dpu-mir-466c & scaffold_30:678246-678266 & UGUGUGUGUGUAUGUGUGUAA \\
\hline dpu-mir-466d & scaffold_67:693500-693521 & GUGUGUGUGUGAGUGUGUGUGU \\
\hline dpu-mir-466e & scaffold_169:10076-10095 & UUGUGUGUGUGAGUGUGUGU \\
\hline dpu-mir-466f & scaffold_6332:737-758 & GUGUGUGUGUGAGUGUGUGAUU \\
\hline dpu-mir-467 & scaffold_397:474-495 & UACAUACACACACAUAUACUAU \\
\hline dpu-mir-5106 & scaffold_76:500686-500706 & GUCGGUAGCUCAGUUGGCAGA \\
\hline dpu-mir-513 & scaffold_54:604492-604513 & ACAGGGAGGUGUCAUUUACUAC \\
\hline dpu-mir-541 & scaffold_3:2756631-2756652 & GGCGAACACAGAAUCCAUUCUU \\
\hline dpu-mir-5416 & scaffold_12:1407447-1407468 & AUUCCUUUUCUCUUUUUUCUUU \\
\hline dpu-mir-574a & scaffold_5:670353-670374 & GUGUGUGUGUGUGAGUGUCGGA \\
\hline dpu-mir-574b & scaffold_5:1791308-1791288 & GUGUGUGUGUGUGAGUGGUGU \\
\hline dpu-mir-638 & scaffold_7427:1389-1410 & AGGGAUCGCGGGCGGGCGGCCC \\
\hline dpu-mir-669 & scaffold_88:40467-40488 & UACACACACACACACAAGUAUU \\
\hline
\end{tabular}




\begin{tabular}{|l|l|l|} 
dpu-mir-79 & scaffold_2:1526251-1526273 & AUAAAGCUAGGUUACCAAAGUUA \\
\hline dpu-mir-7a & scaffold_191:112613-112589 & UGGAAGACUAGUGAUUUUGUUGUUC \\
\hline dpu-mir-7b & scaffold_11571:1094-1070 & UGGAAGACUAGUGAUUUUGUUGUUC \\
\hline dpu-mir-87 & scaffold_1:2191121-2191142 & GUGAGCAAAGUUUCAGGUGCGU \\
\hline dpu-mir-9 & scaffold_2:1526213-1526235 & UCUUUGGUUAUCUAGCUGUAUGA \\
\hline dpu-mir-967 & scaffold_1088:1977-1957 & CUUUUCCACCUAGGUGUUUCU \\
\hline dpu-mir-bantam & scaffold_115:370209-370231 & UGAGAUCAUUGUGAAAGCUGAUU \\
\hline
\end{tabular}

Table S2. Primer sequences for RT-PCR.

\begin{tabular}{|c|c|c|}
\hline \multirow[t]{2}{*}{ mir-1 } & RT primer & GTCGTATCCAGTGCAGGGTCCGAGGTATTCGCACTGGATACGACCTCCAT \\
\hline & $\begin{array}{l}\text { Forward } \\
\text { primer }\end{array}$ & GCGGCGTGGAATGTAAAGAAGT \\
\hline \multirow[t]{2}{*}{ mir-2 } & RT primer & GTCGTATCCAGTGCAGGGTCCGAGGTATTCGCACTGGATACGACGCTCAT \\
\hline & $\begin{array}{l}\text { Forward } \\
\text { primer }\end{array}$ & CACCGACTATCACAGCCAGCTTTG \\
\hline \multirow[t]{2}{*}{ mir-7 } & RT primer & GTCGTATCCAGTGCAGGGTCCGAGGTATTCGCACTGGATACGACACAACA \\
\hline & $\begin{array}{l}\text { Forward } \\
\text { primer }\end{array}$ & GCGGCGTGGAAGACTAGTGATTT \\
\hline \multirow[t]{2}{*}{ mir-8 } & RT primer & GTCGTATCCAGTGCAGGGTCCGAGGTATTCGCACTGGATACGACGACATA \\
\hline & $\begin{array}{l}\text { Forward } \\
\text { primer }\end{array}$ & CGCCGATAATACTGTCAGGTAAA \\
\hline \multirow[t]{2}{*}{ mir-9 } & RT primer & GTCGTATCCAGTGCAGGGTCCGAGGTATTCGCACTGGATACGACTCATAC \\
\hline & $\begin{array}{l}\text { Forward } \\
\text { primer }\end{array}$ & AGCGCTCTTTTGGTTATCTAGCT \\
\hline \multirow[t]{2}{*}{$\operatorname{mir}-10$} & RT primer & GTCGTATCCAGTGCAGGGTCCGAGGTATTCGCACTGGATACGACACAAAT \\
\hline & $\begin{array}{l}\text { Forward } \\
\text { primer }\end{array}$ & CGTGAATGGGACATCTAGGCT \\
\hline \multirow[t]{2}{*}{ mir-12 } & RT primer & GTCGTATCCAGTGCAGGGTCCGAGGTATTCGCACTGGATACGACACCAGT \\
\hline & $\begin{array}{l}\text { Forward } \\
\text { primer }\end{array}$ & ACGGCTGAGTATTACATCAGGT \\
\hline \multirow[t]{2}{*}{ mir-92 } & RT primer & GTCGTATCCAGTGCAGGGTCCGAGGTATTCGCACTGGATACGACGCAGGC \\
\hline & $\begin{array}{l}\text { Forward } \\
\text { primer }\end{array}$ & GGATCAATTGCACTCGTCCCG \\
\hline \multirow[t]{2}{*}{ mir-100 } & RT primer & GTCGTATCCAGTGCAGGGTCCGAGGTATTCGCACTGGATACGACACACAA \\
\hline & $\begin{array}{l}\text { Forward } \\
\text { primer }\end{array}$ & TGACCAACCCGTAGATCCGAAC \\
\hline \multirow[t]{2}{*}{ mir-133 } & RT primer & GTCGTATCCAGTGCAGGGTCCGAGGTATTCGCACTGGATACGACACAGCT \\
\hline & $\begin{array}{l}\text { Forward } \\
\text { primer }\end{array}$ & GCATGAACCAGGGGAAGTTGG \\
\hline \multirow[t]{2}{*}{ mir-153 } & RT primer & GTCGTATCCAGTGCAGGGTCCGAGGTATTCGCACTGGATACGACCATCAC \\
\hline & $\begin{array}{l}\text { Forward } \\
\text { primer }\end{array}$ & CGCGATTGCATAGTCACAAAA \\
\hline
\end{tabular}




\begin{tabular}{|c|c|c|}
\hline \multirow[t]{2}{*}{ mir-283 } & RT primer & GTCGTATCCAGTGCAGGGTCCGAGGTATTCGCACTGGATACGACAGAATT \\
\hline & $\begin{array}{l}\text { Forward } \\
\text { primer }\end{array}$ & GCGCGAAATATCAGCAGGT \\
\hline \multirow[t]{2}{*}{$\operatorname{mir}-71$} & RT primer & GTCGTATCCAGTGCAGGGTCCGAGGTATTCGCACTGGATACGACCATCTC \\
\hline & $\begin{array}{l}\text { Forward } \\
\text { primer }\end{array}$ & GCGGCGTGAAAGACATGGGTAGT \\
\hline \multirow[t]{2}{*}{$\operatorname{mir}-33$} & RT primer & GTCGTATCCAGTGCAGGGTCCGAGGTATTCGCACTGGATACGACGTGCAA \\
\hline & $\begin{array}{l}\text { Forward } \\
\text { primer }\end{array}$ & GCGGCGGTGCATTGTAGTTGCA \\
\hline \multirow[t]{2}{*}{$\operatorname{mir}-34$} & RT primer & GTCGTATCCAGTGCAGGGTCCGAGGTATTCGCACTGGATACGACCACAAC \\
\hline & $\begin{array}{l}\text { Forward } \\
\text { primer }\end{array}$ & GTCGACTGGCAGTGTGGTTAGCTG \\
\hline \multirow[t]{2}{*}{ mir-317 } & RT primer & GTCGTATCCAGTGCAGGGTCCGAGGTATTCGCACTGGATACGACACTGAG \\
\hline & $\begin{array}{l}\text { Forward } \\
\text { primer }\end{array}$ & GCGGCGTGAACACAGCTGGTGGTAT \\
\hline \multirow[t]{2}{*}{ mir-204 } & RT primer & GTCGTATCCAGTGCAGGGTCCGAGGTATTCGCACTGGATACGACGGTCCC \\
\hline & $\begin{array}{l}\text { Forward } \\
\text { primer }\end{array}$ & GAGGCCGTGTGCGTGTGACAGC \\
\hline \multirow[t]{2}{*}{ mir-210 } & RT primer & GTCGTATCCAGTGCAGGGTCCGAGGTATTCGCACTGGATACGACTCAGCC \\
\hline & $\begin{array}{l}\text { Forward } \\
\text { primer }\end{array}$ & GGGCGCCTTGTGCGTGTGACAGC \\
\hline \multirow[t]{2}{*}{$\operatorname{mir}-279$} & RT primer & $\begin{array}{l}\text { GTCGTATCCAGTGCAGGGTCCGAGGTATTCGCACTGGATACGACTGGATG } \\
\end{array}$ \\
\hline & $\begin{array}{l}\text { Forward } \\
\text { primer }\end{array}$ & GCGCGCTGACTAGATCCACACT \\
\hline \multirow[t]{3}{*}{ mir-7444 } & RT primer & GTCGTATCCAGTGCAGGGTCCGAGGTATTCGCACTGGATACGACGGAGGT \\
\hline & $\begin{array}{l}\text { Forward } \\
\text { primer }\end{array}$ & GCGCGCGTAAGGCTCCTTCTG \\
\hline & $\begin{array}{l}\text { Universal } \\
\text { Reverse } \\
\text { Primer }\end{array}$ & GTGCAGGGTCCGAGGT \\
\hline \multirow[t]{2}{*}{ SCN2A } & $\begin{array}{l}\text { Forward } \\
\text { primer }\end{array}$ & GAAACGGAGCCAGTTACCAA \\
\hline & $\begin{array}{l}\text { Reverse } \\
\text { primer }\end{array}$ & GAGAGGGGTTTTGGGTGATT \\
\hline \multirow[t]{2}{*}{ SLC31A1 } & $\begin{array}{l}\text { Forward } \\
\text { primer }\end{array}$ & TGCAAATTTGGACGTCTTCA \\
\hline & $\begin{array}{l}\text { Reverse } \\
\text { primer }\end{array}$ & TGGGGGATTCAAGATGCTAC \\
\hline \multirow[t]{2}{*}{ KCNN1 } & $\begin{array}{l}\text { Forward } \\
\text { primer }\end{array}$ & AACGGTATCACGACGAGGAG \\
\hline & $\begin{array}{l}\text { Reverse } \\
\text { primer }\end{array}$ & TCCAATTTCCTCGAGACGAC \\
\hline
\end{tabular}


Table S3. Differentially expressed (fold change of $>1.5$; FDR $<0.05$ ) miRNAs after exposure of Daphnia pulex nenonates to low $(20 \mu \mathrm{g} / \mathrm{L})$ or high $(40 \mu \mathrm{g} / \mathrm{L})$ cadmium chloride $\left(\mathrm{CdCl}_{2}\right)$ for $48 \mathrm{~h}$. Orange color represent up-regulated miRNAs and green color represent down-regulated miRNAs. AE is the average expression of control, Cd-low and Cd-high group.

\begin{tabular}{|c|c|c|c|}
\hline Cd Exposure & miRNA name & Fold Change & $\log _{2} A E$ \\
\hline \multirow{22}{*}{ Cd-Low } & miR-71 & 1.61 & 12.49 \\
\hline & miR-92 & 2.85 & 2.59 \\
\hline & miR-204 & 3.50 & 2.43 \\
\hline & miR-210 & 4.85 & 4.89 \\
\hline & miR-252 & 2.93 & 7.16 \\
\hline & miR-279 & 7.19 & 5.67 \\
\hline & miR-615 & 2.79 & 2.61 \\
\hline & miR-871 & 2.98 & 2.51 \\
\hline & miR-1076 & 2.43 & 3.37 \\
\hline & miR-1414 & 2.01 & 2.25 \\
\hline & miR-1894 & 2.88 & 2.42 \\
\hline & miR-2219 & 2.20 & 2.42 \\
\hline & miR-4259 & 1.71 & 2.09 \\
\hline & miR-6831 & 2.28 & 2.29 \\
\hline & miR-7422 & 2.07 & 3.13 \\
\hline & miR-7666 & 1.79 & 2.01 \\
\hline & miR-217 & -1.72 & 1.51 \\
\hline & miR-344 & -1.86 & 1.94 \\
\hline & miR-345 & -3.33 & 2.13 \\
\hline & miR-6743 & -1.92 & 8.23 \\
\hline & miR-7444 & -2.37 & 1.92 \\
\hline & miR-7653- & -2.00 & 3.24 \\
\hline \multirow{13}{*}{ Cd-High } & miR-71 & 1.61 & 12.49 \\
\hline & miR-194 & 1.75 & 2.02 \\
\hline & miR-204 & 2.51 & 2.43 \\
\hline & miR-210 & 1.50 & 11.14 \\
\hline & miR-252 & 2.47 & 7.80 \\
\hline & miR-278 & 2.48 & 1.98 \\
\hline & miR-279 & 3.72 & 5.67 \\
\hline & miR-615 & 2.63 & 2.58 \\
\hline & miR-1076 & 1.61 & 3.37 \\
\hline & miR-1414 & 2.04 & 2.25 \\
\hline & miR-2219 & 1.54 & 2.42 \\
\hline & miR-2441 & 2.16 & 2.09 \\
\hline & miR-6831 & 2.42 & 2.29 \\
\hline
\end{tabular}




\begin{tabular}{|l|l|r|r|}
\hline & miR-144 & -1.80 & 1.71 \\
\cline { 2 - 4 } & miR-216 & -1.65 & 1.65 \\
\cline { 2 - 4 } & miR-344 & -1.84 & 1.94 \\
\cline { 2 - 4 } & miR-345 & -2.85 & 2.13 \\
\cline { 2 - 4 } miR-6743 & -1.98 & 8.23 \\
\cline { 2 - 4 } & miR-7444 & -1.81 & 1.92 \\
\cline { 2 - 4 } & miR-7670 & -1.67 & 2.07 \\
\cline { 2 - 4 } & miR-7939 & -1.54 & 1.97 \\
\hline
\end{tabular}


Table S4. Robustly expressed miRNAs, Prob ID on the Affymetrix Genechip 4.0 array, sequence of mature miRNA and genome loci of mature miRNAs. Letter $\mathrm{Y}$ denotes miRNAs predicted by Chen et al. (2014) ${ }^{25}$ and letter $\mathbf{N}$ denotes miRNAs that are newly identified by microarray.

\begin{tabular}{|c|c|c|c|c|}
\hline miRNA Name & Prob ID & miRNA Sequence & Genome Loci & \\
\hline dpu-bantam & MIMAT0012634_st & UGAGAUCAUUGUGAAAGCUGAUU & scaffold_115:370209-370231 & $\mathrm{Y}$ \\
\hline dpu-let-7 & MIMAT0001761_st & UGAGGUAGUAGGUUGUAUGGUU & scaffold_71:446521-446500 & $\mathrm{N}$ \\
\hline dpu-miR-1 & MIMAT0001199_st & UGGAAUGUAAAGAAGUAUGGAG & scaffold_1:1720944-1720923 & $\mathrm{Y}$ \\
\hline dpu-miR-2 & MIMAT0012657_st & UAUCACAGCCAGCUUUGAUGAGC & scaffold_80:240622-240644 & $\mathrm{Y}$ \\
\hline dpu-miR-7 & MIMAT0012666_st & UGGAAGACUAGUGAUUUUGUUGU & scaffold_191:112613-112589 & $\mathrm{Y}$ \\
\hline dpu-miR-8 & MIMAT0012669_st & UAAUACUGUCAGGUAAAGAUGUC & scaffold_131:139446-139468 & $\mathrm{Y}$ \\
\hline dpu-miR-10 & MIMAT0012636_st & UACCCUGUAGAUCCGAAUUUGU & scaffold_7:304885-304864 & $\mathrm{Y}$ \\
\hline dpu-miR-12 & MIMAT0012638_st & UGAGUAUUACAUCAGGUACUGGU & scaffold_1:1847905-1847883 & $\mathrm{Y}$ \\
\hline dpu-miR-31 & MIMAT0012659_st & AGGCAAGAUGUCGGCAUAGCUGA & scaffold_121:73946-73960 & $\mathrm{N}$ \\
\hline dpu-miR-34 & MIMAT0012663_st & UGGCAGUGUGGUUAGCUGGUUGUG & scaffold_4:1242114-1242091 & $\mathrm{Y}$ \\
\hline dpu-miR-71 & MIMAT0012667_st & UGAAAGACAUGGGUAGUGAGAUG & scaffold_80:240430-240452 & $\mathrm{Y}$ \\
\hline dpu-miR-72 & MIMAT0014107_st & AGGCAAGAUGUUGGCAUAGCUGA & scaffold_250:89868-89850 & $\mathrm{N}$ \\
\hline dpu-miR-87 & MIMAT0011644_st & GUGAGCAAAGUUUCAGGUGUGC & scaffold_1:2190959-2190977 & $\mathrm{N}$ \\
\hline dpu-miR-92 & MIMAT0012672_st & AAUUGCACUCGUCCCGGCCUGC & scaffold_38:876194-876215 & $\mathrm{Y}$ \\
\hline dpu-miR-99 & MIMAT0001812_st & AACCCGUAGAUCCGAUCUUGUG & scaffold_71:446720-446699 & $\mathrm{N}$ \\
\hline dpu-miR-100 & MIMAT0012637_st & AACCCGUAGAUCCGAACUUGUGU & scaffold_71:446720-446698 & $\mathrm{N}$ \\
\hline dpu-miR-124 & MIMAT0011541_st & UAAGGCACGCGGUGAAUGCC & scaffold_120:76940-76959 & $\mathrm{N}$ \\
\hline dpu-miR-125 & MIMAT0001230_st & UCCCUGAGACCCUAACUUGUGA & scaffold_71:445406-445427 & $\mathrm{Y}$ \\
\hline dpu-miR-129 & MIMAT0003035_st & CUUUUUGCGGUCUGGGCUUGCU & scaffold_15241:831-850 & $\mathrm{N}$ \\
\hline dpu-miR-133 & MIMAT0012640_st & UUGGUCCCCUUCAACCAGCUGU & scaffold_1:1708478-1708457 & $\mathrm{Y}$ \\
\hline
\end{tabular}




\begin{tabular}{|c|c|c|c|c|}
\hline $149-3 p$ & MIMAT0026827_st & GAGGGAGGGACGGGGGCUGUGC & scaffold_44:232313-232333 & $\mathrm{N}$ \\
\hline dpu-miR-150-3p & MIMAT0007091_st & CCUGGUACGGGGCCGUGGGAGAG & scaffold_373:12654-12641 & $\mathrm{N}$ \\
\hline dpu-miR-182 & MIMAT0009529_st & CUUGGCACUGGUAGAAUUCACU & scaffold_87:475817-475837 & $\mathrm{N}$ \\
\hline dpu-miR-183 & MIMAT0009584_st & AAUGGCACUGGUAGAAUUCACGG & scaffold_87:475620-475642 & $\mathrm{N}$ \\
\hline dpu-miR-184 & MIMAT0001232_st & UGGACGGAGAACUGAUAAGGGC & scaffold_24:317115-317138 & $\mathrm{Y}$ \\
\hline dpu-miR-193 & MIMAT0012643_st & UACUGGCCUGCUAAGUCCCAAA & scaffold_167:85478-85457 & $\mathrm{N}$ \\
\hline dpu-miR-198 & MIMAT0000228_st & GGUCCAGAGGGGAGAUAGGUUC & scaffold_138:122426-122440 & $\mathrm{N}$ \\
\hline dpu-miR-210 & MIMAT0007903_st & UUGUGCGUGUGACAGCGGCU & scaffold_51:480364-480342 & $\mathrm{Y}$ \\
\hline dpu-miR-252 & MIMAT0011649_st & CUAAGUAGUAGUGCCGCAGGU & scaffold_8:127380-127400 & $\mathrm{N}$ \\
\hline dpu-miR-263a & MIMAT0012649_st & AAUGGCACUGGAAGAAUUCAC & scaffold_87:475620-475643 & $\mathrm{Y}$ \\
\hline dpu-miR-275 & MIMAT0012651_st & UCAGGUACCUGAAGUAGCGCGCG & scaffold_4:1790782-1790804 & $\mathrm{N}$ \\
\hline dpu-miR-276 & MIMAT0012652_st & UAGGAACUUCAUACCGUGCUCU & scaffold_15:755667-755688 & $\mathrm{N}$ \\
\hline dpu-miR-278 & MIMAT0012653_st & UCGGUGGGACUUUCGUCCGUGU & 60:694726-694705 & $\mathrm{N}$ \\
\hline dpu-miR-279 & MIMAT0009539_st & UGACUAGAUCCACACUCAUCC & scaffold_63:523674-523654 & $\mathrm{N}$ \\
\hline dpu-miR-281 & MIMAT0012655_st & UGUCAUGGAGCUGCUCUCUUUAU & scaffold_11:1065371-1065349 & $\mathrm{N}$ \\
\hline dpu-miR-285 & MIMAT0012656_st & UAGCACCAUUGGAAUUCAGUUU & scaffold_1:332555-332576 & $\mathrm{N}$ \\
\hline dpu-miR-289 & MIMAT0001253_st & UAAAUAUUUAAGUGGAGCCUGCGACU & scaffold_193:101255-101240 & $\mathrm{N}$ \\
\hline dpu-miR-296 & MIMAT0023880_st & GAGGGUUGGGUGGAGGCUCU & scaffold_5:1975951-1975966 & $\mathrm{N}$ \\
\hline dpu-miR-305 & MIMAT0001255_st & AUUGUACUUCAUCAGGUGCUCUG & scaffold_4:1791164-1791187 & $\mathrm{Y}$ \\
\hline dpu-miR-315 & MIMAT0009541_st & UUUUGAUUGUUGCUCAGAAAGCC & scaffold_58:431908-431930 & $\mathrm{Y}$ \\
\hline dpu-miR-317 & MIMAT0001488_st & UGAACACAGCUGGUGGUAUCUCAGU & scaffold_4:1243985-1243961 & $\mathrm{N}$ \\
\hline dpu-miR-320 & MIMAT0013878_st & AAAAGCUGGGUUGAGAGGGCGAA & scaffold_13:1519265-1519283 & $\mathrm{N}$ \\
\hline dpu-miR-335 & MIMAT0013955_st & UCAAGAGCAAUAACGAAAAAUG & scaffold_286:83572-83553 & $\mathrm{N}$ \\
\hline dpu-miR-371 & MIMAT0007747_st & ACUCAAAAAAUGGCGGCACUUU & scaffold_1364:4113-4127 & $\mathrm{N}$ \\
\hline
\end{tabular}




\begin{tabular}{|c|c|c|c|c|}
\hline 75 & MIMAT0012664_st & UUUGUUCGUUCGGCUUGAGUUA & scaffold_55:302090-302111 & $\mathrm{Y}$ \\
\hline dpu-miR-455 & MIMAT0008157_st & GCAGUCCAUGGGCAUAUACAC & scaffold_2163:1093-1113 & $\mathrm{N}$ \\
\hline dpu-miR-484 & MIMAT0017974_st & CCCAGGGGGCGACCCAGGCU & scaffold_6:825679-825692 & $\mathrm{N}$ \\
\hline dpu-miR-494 & MIMAT0008168_st & UGAAACAUACACGGGAAACCUC & scaffold_8:241979-241962 & $\mathrm{N}$ \\
\hline dpu-miR-557 & MIMAT0008238_st & GUUUGCACGGGUGGGCCUUGUCU & scaffold_776:8494-8507 & $\mathrm{N}$ \\
\hline dpu-miR-564 & MIMAT0016009_st & AGGCACGGUGGCAGCAGGC & scaffold_1621:4132-4116 & $\mathrm{N}$ \\
\hline dpu-miR-604 & MIMAT0006459_st & GGCUGCGGGAUUCAGGAC & scaffold_36:946054-946040 & $\mathrm{N}$ \\
\hline dpu-miR-606 & MIMAT0012810_st & AAACUACUAAAAAUCAAAGAU & scaffold_108:297986-298000 & $\mathrm{N}$ \\
\hline dpu-miR-637 & MIMAT0008293_st & ACUGGGGGCUUUCGGGCUCUGCGU & scaffold_11556:969-951 & $\mathrm{N}$ \\
\hline dpu-miR-638 & MIMAT0003308_st & AGGGAUCGCGGGCGGGUGGCGGCCU & scaffold_7427:1389-1408 & $\mathrm{N}$ \\
\hline dpu-miR-658 & MIMAT0009362_st & GGCGGAGGGAAGCGGGUCCGUUGGU & scaffold_573:951-964 & $\mathrm{N}$ \\
\hline dpu-miR-663 & MIMAT0006502_st & AGGCGGGGCGCUGCGGGACCGC & scaffold_7427:1389-1410 & $\mathrm{Y}$ \\
\hline dpu-miR-678 & MIMAT0012857_st & GUCUCGGGGCAAGGACUGGAGG & scaffold_11:1703007-1702988 & $\mathrm{N}$ \\
\hline dpu-miR-705 & MIMAT0003495_st & GGUGGGAGGUGGGGUGGGCA & scaffold_12549:258-273 & $\mathrm{N}$ \\
\hline dpu-miR-706 & MIMAT0003496_st & AGAGAAACCCUGUCUCAAAAAA & scaffold_72:301047-301064 & $\mathrm{N}$ \\
\hline dpu-miR-709 & MIMAT0003499_st & GGAGGCAGAGGCAGGAGGA & scaffold_16:1068672-1068657 & $\mathrm{N}$ \\
\hline dpu-miR-714 & MIMAT0003505_st & CGACGAGGGCCGGUCGGUCGC & scaffold_8:1126164-1126147 & $\mathrm{N}$ \\
\hline dpu-miR-737 & MIMAT0003768_st & AAUCAAAACCUAAAGAAAAUA & scaffold_20:883696-883712 & $\mathrm{N}$ \\
\hline dpu-miR-745 & MIMAT0012646_st & GAGCUGCCCAGUGAAGGGCUUU & scaffold_63:362018-361997 & $\mathrm{N}$ \\
\hline dpu-miR-762 & MIMAT0012791_st & GGGGCUGGGGCCGGAGCCGAGC & scaffold_14:1356104-1356086 & $\mathrm{N}$ \\
\hline dpu-miR-877 & MIMAT0024155_st & GUAGAGGAGAUGGCGCAGGGGACA & scaffold_4:290034-290050 & $\mathrm{N}$ \\
\hline dpu-miR-933 & MIMAT0004976_st & UGUGCGCAGGGAGACCUCUCCC & scaffold_21:774461-774446 & $\mathrm{N}$ \\
\hline dpu-miR-940 & MIMAT0024213_st & AAGGCAGGGCCCCCGCUCCCC & scaffold_29:31181-31194 & $\mathrm{N}$ \\
\hline dpu-miR-965 & MIMAT0012674_st & UAAGCGUAUGGCUUUUCCCCUG & scaffold_32:27803-27782 & $\mathrm{N}$ \\
\hline
\end{tabular}




\begin{tabular}{|c|c|c|c|c|}
\hline dpu-miR-981 & MIMAT0010098_st & UUCGUUGUCGACGAAACCUGCA & scaffold_2:1451012-1450991 & $Y$ \\
\hline dpu-miR-993 & MIMAT0025000_st & GAAGCUCGUCUCUACAGGUAUCU & scaffold_7:282360-282382 & $Y$ \\
\hline dpu-miR-1000-3p & MIMAT0020900_st & CUGCUGGGUCGGGGCAUUAACA & scaffold_45:205959-205946 & $\mathrm{N}$ \\
\hline dpu-miR-1224 & MIMAT0012827_st & GUGAGGACUGGGGAGGUGGAG & scaffold_112:87036-87051 & $\mathrm{N}$ \\
\hline dpu-miR-1227 & MIMAT0005581_st & GUGGGGCCAGGCGGUGGUGG & scaffold_26:997417-997403 & $\mathrm{N}$ \\
\hline dpu-miR-1228 & MIMAT0024229_st & GUGGGCGGGGGCAGGUGUGUGG & scaffold_197:58182-58195 & $\mathrm{N}$ \\
\hline dpu-miR-1260 & MIMAT0023746_st & AUCCCACCGCUGCCACCA & scaffold_9:1110005-1110021 & $\mathrm{N}$ \\
\hline dpu-miR-1268 & MIMAT0016192_st & CGGGCGUGGUGGUGGGCG & scaffold_47:67803-67789 & $\mathrm{N}$ \\
\hline dpu-miR-1281 & MIMAT0005939_st & UCGCCUCCUCCUCUCCC & scaffold_21:1417345-1417329 & $\mathrm{N}$ \\
\hline dpu-miR-1346 & MIMAT0006919_st & GUGGGUUGGGGGCGGGGG & scaffold_138:198762-198779 & $\mathrm{N}$ \\
\hline dpu-miR-1383 & MIMAT0007131_st & UUGGUCCCCUUCCCCCCG & scaffold_142:129306-129322 & $\mathrm{N}$ \\
\hline dpu-miR-1394 & MIMAT0007196_st & CCCGGGGCGCCCGAGGGG & & $\mathrm{N}$ \\
\hline dpu-miR-1412 & MIMAT0007232_st & UGGGUGAGGAGGGCGAGG & scaffold_3:2242631-2242617 & $\mathrm{N}$ \\
\hline dpu-miR-1469 & MIMAT0007347_st & CUCGGCGCGGGGCGCGGGCUCC & scaffold_9:1777440-1777426 & $\mathrm{N}$ \\
\hline dpu-miR-1576 & MIMAT0007437_st & CUCGGGCGGGCGGAGCGGCAC & scaffold_77:150720-150736 & $\mathrm{N}$ \\
\hline dpu-miR-1579 & MIMAT0007440_st & CGUGGCGGGAAGGGAUGGGCG & scaffold_47:34351-34334 & $\mathrm{N}$ \\
\hline dpu-miR-1584 & MIMAT0007445_st & CCGGGUGGGGCUGGGCUGGG & scaffold_43:735422-735407 & $\mathrm{N}$ \\
\hline dpu-miR-1587 & MIMAT0007449_st & GGGCUGGGCUGGGCUGGGCA & scaffold_252:76011-76029 & $\mathrm{N}$ \\
\hline dpu-miR-1594 & MIMAT0007457_st & UGGUGGGGAUGGGUUGGGGUU & scaffold_4221:94-77 & $\mathrm{N}$ \\
\hline dpu-miR-1599 & MIMAT0007465_st & GGAGGGAGGAAAAAAAAAAA & scaffold_74:196968-196986 & $\mathrm{N}$ \\
\hline dpu-miR-1607 & MIMAT0007473_st & AGGGGCGGGAGGGGUCGGGC & scaffold_118:111571-111558 & $\mathrm{N}$ \\
\hline dpu-miR-1610 & MIMAT0007476_st & UGGCUUGUGGUGGAACGGGCG & scaffold_138:297175-297189 & $\mathrm{N}$ \\
\hline dpu-miR-1631 & MIMAT0007502_st & AAACUGGCGACGUGGGACGGUC & scaffold_16:1636728-1636714 & $\mathrm{N}$ \\
\hline dpu-miR-1636 & MIMAT0007509_st & UGCAGGUGAUGGCGGGGCUG & scaffold_90:296946-296960 & $\mathrm{N}$ \\
\hline dpu-miR-1694 & MIMAT0007586_st & GGAGGACGAGGCUGCGAGCGGA & scaffold_221:83596-83610 & $\mathrm{N}$ \\
\hline
\end{tabular}




\begin{tabular}{|c|c|c|c|c|}
\hline dpu-miR-1716 & MIMAT0007613_st & AGCGGGGCGGCUGUGAGCUGAGCU & scaffold_88:119562-119547 & $\mathrm{N}$ \\
\hline dpu-miR-1763 & MIMAT0007670_st & GAGGGCGGGAGCGGAGCGCGGGA & scaffold_41:230029-230046 & $\mathrm{N}$ \\
\hline dpu-miR-1770 & MIMAT0007679_st & CUGCGGGGGAGGAGGGAGGGGG & scaffold_128:139414-139396 & $\mathrm{N}$ \\
\hline dpu-miR-1777 & MIMAT0007688_st & GUGGGCGGUGCGGGGCGGCG & scaffold_619:6053-6039 & $\mathrm{N}$ \\
\hline dpu-miR-1790 & MIMAT0007703_st & CGGGUGAGGGCGGUGGGGGA & scaffold_1:1135821-1135806 & $\mathrm{N}$ \\
\hline dpu-miR-1825 & MIMAT0006765_st & UCCAGUGCCCUCCUCUCC & scaffold_419:31089-31105 & $\mathrm{N}$ \\
\hline dpu-miR-1844 & MIMAT0006740_st & AGGACUACGGACGGGCUGAG & scaffold_1443:992-979 & $\mathrm{N}$ \\
\hline dpu-miR-1892 & MIMAT0007871_st & AUUUGGGGACGGGAGGGAGGAU & scaffold_5:1894963-1894949 & $\mathrm{N}$ \\
\hline dpu-miR-1892 & MIMAT0012895_st & AUUUGGGGUGGGGGAUGGGGA & scaffold_5:1894963-1894949 & $\mathrm{N}$ \\
\hline dpu-miR-1895 & MIMAT0007867_st & CCCCCGAGGAGGACGAGGAGGA & scaffold_245:33553-33570 & $\mathrm{N}$ \\
\hline dpu-miR-1908 & MIMAT0016237_st & CGGCGGGGAAGGCGAUUGGUC & scaffold_6:505253-505238 & $\mathrm{N}$ \\
\hline dpu-miR-1915 & MIMAT0016243_st & CCCCAGGGCGACGCGGCGGG & scaffold_10032:1077-1063 & $\mathrm{N}$ \\
\hline dpu-miR-1926a & MIMAT0024494_st & AGGAAUUCUAAAGCAAAAA & scaffold_6080:2080-2065 & $\mathrm{N}$ \\
\hline dpu-miR-1926b & MIMAT0007933_st & AGGAAUUCUAAAGCAAAAAGG & scaffold_172:145886-145871 & $\mathrm{N}$ \\
\hline dpu-miR-1931 & MIMAT0009394_st & AUGCAAGGGCUGGUGCGAUGGC & scaffold_12352:982-968 & $\mathrm{N}$ \\
\hline dpu-miR-2126 & MIMAT0011202_st & AGCGGCGCGGUAGGAGCA & scaffold_40:283141-283122 & $\mathrm{N}$ \\
\hline dpu-miR-2127 & MIMAT0011203_st & CGGGAGGGGAGGGAGGGCGGG & scaffold_40:283141-283122 & $\mathrm{N}$ \\
\hline dpu-miR-2137 & MIMAT0011213_st & GCCGGCGGGAGCCCCAGGGAG & scaffold_99:225700-225716 & $\mathrm{N}$ \\
\hline dpu-miR-2287 & MIMAT0011794_st & CUGGGACUGGUGGCAGCACUU & scaffold_68:188557-188543 & $\mathrm{N}$ \\
\hline dpu-miR-2305 & MIMAT0011817_st & CGGGGGUGGCGGGGAGGGGG & scaffold_5:402732-402716 & $\mathrm{N}$ \\
\hline dpu-miR-2309 & MIMAT0011821_st & UGUGAGGGUGGUGGACGGCAGGG & scaffold_97:212567-212553 & $\mathrm{N}$ \\
\hline dpu-miR-2326 & MIMAT0011855_st & CCCCCCUUCCUCUGGAAAAA & scaffold_5:1650448-1650430 & $\mathrm{N}$ \\
\hline dpu-miR-2343 & MIMAT0011878_st & AAGGGGAGACGGUGGAACUUAU & scaffold_55:147880-147899 & $\mathrm{N}$ \\
\hline dpu-miR-2348 & MIMAT0011883_st & UUCGGGUGGUGUGGAGCGGCC & scaffold_2:3635653-3635667 & $\mathrm{N}$ \\
\hline dpu-miR-2360 & MIMAT0011898_st & CGGGCAGGAGGCUGGAGGCGUU & scaffold_65:410930-410915 & $\mathrm{N}$ \\
\hline
\end{tabular}




\begin{tabular}{|c|c|c|c|c|}
\hline dpu-miR-2374 & MIMAT0011920_st & UUGGGGCUGGGGAGAGGCGGG & scaffold_21:1277716-1277699 & $\mathrm{N}$ \\
\hline dpu-miR-2389 & MIMAT0011942_st & GAGGGGACUCGGGAGUCGGAG & scaffold_4739:3372-3386 & $\mathrm{N}$ \\
\hline dpu-miR-2392 & MIMAT0011945_st & AUGGAUGGGGGUGAGGGGUGCA & scaffold_175:207735-207749 & $\mathrm{N}$ \\
\hline dpu-miR-2407 & MIMAT0011965_st & CUGGGCGGAUGGGAAGGGCUGG & scaffold_101:300649-300663 & $\mathrm{N}$ \\
\hline dpu-miR-2411 & MIMAT0025389_st & UGGAGUGACUGUCAGAUGCAGC & scaffold_76:611455-611442 & $\mathrm{N}$ \\
\hline dpu-miR-2412 & MIMAT0011974_st & UUGGGGCUGGGUGGGGAGAGGG & scaffold_6:1859246-1859265 & $\mathrm{N}$ \\
\hline dpu-miR-2418 & MIMAT0011982_st & UGGGAUGAGUGUGGCGUGGUCG & scaffold_48:303489-303476 & $\mathrm{N}$ \\
\hline dpu-miR-2422 & MIMAT0011989_st & UUGAGGGGACUGAGGUGCGGAG & scaffold_134:136004-135991 & $\mathrm{N}$ \\
\hline dpu-miR-2428 & MIMAT0011998_st & UGGCUGGAGUGCGGCGGAGGU & scaffold_1505:2486-2473 & $\mathrm{N}$ \\
\hline dpu-miR-2442 & MIMAT0012017_st & AGAGCAGGGGCUGUGGGCUGCA & scaffold_17:284673-284659 & $\mathrm{N}$ \\
\hline dpu-miR-2449 & MIMAT0012027_st & UGGGCAGGAGACAGCAGGGC & scaffold_43:386943-386959 & $\mathrm{N}$ \\
\hline dpu-miR-2455 & MIMAT0012037_st & UCUGUGCUCGGGGAGGCAGGGA & scaffold_395:84-97 & $\mathrm{N}$ \\
\hline dpu-miR-2478 & MIMAT0012070_st & GUAUCCCACUUCUGACACCA & scaffold_41:85012-85031 & $\mathrm{N}$ \\
\hline dpu-miR-2487 & MIMAT0012083_st & CUCUAAGGGCUGGGCCGGUCGG & scaffold_20:456178-456164 & $\mathrm{N}$ \\
\hline dpu-miR-2518 & MIMAT0012301_st & UCGAGCAGCGGGUCGAUCCGAGC & scaffold_59:209946-209931 & $\mathrm{N}$ \\
\hline dpu-miR-2776 & MIMAT0013660_st & AGAGUACGCAAAAAAACAAUU & scaffold_64:532302-532317 & $\mathrm{N}$ \\
\hline dpu-miR-2779 & MIMAT0013668_st & AUAUCCGGCUCGAAGGACCA & scaffold_1861:1195-1179 & $\mathrm{N}$ \\
\hline dpu-miR-2783 & MIMAT0013679_st & CGGGCGAGGGUGUGGGUGUGGCG & scaffold_11:652383-652398 & $\mathrm{N}$ \\
\hline dpu-miR-2861 & MIMAT0013803_st & GGGGCCUGGCGGCGGGCGG & scaffold_2805:6464-6477 & $\mathrm{N}$ \\
\hline dpu-miR-2881 & MIMAT0013839_st & GGGGCGGGGGGGCGGGUG & scaffold_11893:162-179 & $\mathrm{N}$ \\
\hline dpu-miR-2887 & MIMAT0013845_st & CGGGACCGGGGUCCGGUGCG & scaffold_30:335875-335889 & $\mathrm{N}$ \\
\hline dpu-miR-2888 & MIMAT0013846_st & GGUGGGGUGGGGGGGUUGG & scaffold_149:246923-246906 & $\mathrm{N}$ \\
\hline dpu-miR-2892 & MIMAT0013850_st & GGCGACGGAGGCGCGACCCCCC & scaffold_4:1075877-1075891 & $\mathrm{N}$ \\
\hline dpu-miR-2893 & MIMAT0013851_st & GUGGAGGAGAAUGCCCGGGG & scaffold_1657:4629-4643 & $\mathrm{N}$ \\
\hline dpu-miR-2898 & MIMAT0013856_st & UGGUGGAGAUGCCGGGGA & scaffold_155:265410-265424 & $\mathrm{N}$ \\
\hline
\end{tabular}




\begin{tabular}{|c|c|c|c|c|}
\hline dpu-miR- & MIMAT0013857_st & AGGCGGGCCGGGGUUGGA & scaffold_43:142277-142260 & $\mathrm{N}$ \\
\hline dpu-miR-2900 & MIMAT0013858_st & AGGGCGGGCGGCGACUGGAA & scaffold_2:1228200-1228216 & $\mathrm{N}$ \\
\hline dpu-miR-2946 & MIMAT0014325_st & UAGUACGGAAAAGAUAUGGGGA & scaffold_195:142006-141991 & $\mathrm{N}$ \\
\hline dpu-miR-2961 & MIMAT0014461_st & AGGAAGGUAGGGCGGGCGUCGCU & scaffold_8:1789133-1789116 & $\mathrm{N}$ \\
\hline tgu-miR-2966 & MIMAT0014473_st & CCGGGACGGGACCGGGACGGG & scaffold_42:371363-371343 & $\mathrm{N}$ \\
\hline tgu-miR-2974 & MIMAT0014484_st & GCGGGAUGGGCUGGGCCGGG & scaffold_93:334921-334903 & $\mathrm{N}$ \\
\hline dpu-miR-2976 & MIMAT0014486_st & GCGGAGCGGAGCGGAGCGGGG & scaffold_3421:693-675 & $\mathrm{N}$ \\
\hline dpu-miR-2978 & MIMAT0014488_st & GUGGGCUGGGGAAGGGCUGCUGGGU & scaffold_7279:1264-1243 & $\mathrm{N}$ \\
\hline dpu-miR-2981 & MIMAT0014491_st & CCGGGCCGGGCGGGCGGG & scaffold_5:554981-554996 & $\mathrm{N}$ \\
\hline dpu-miR-3010 & MIMAT0014678_st & UGAGAGCGCAAAAAUAAAUUUG & scaffold_8:1334337-1334321 & $\mathrm{N}$ \\
\hline dpu-miR-3049 & MIMAT0014789_st & UCGGGAAGGCAGCUGCGGCGGACU & scaffold_1:529495-529475 & $\mathrm{N}$ \\
\hline dpu-miR-3128 & MIMAT0014991_st & UCUGGCAAGUAAAAAACUCUCAU & scaffold_10:478189-478173 & $\mathrm{N}$ \\
\hline dpu-miR-3141 & MIMAT0024573_st & GAGGGCGGGUGGAGGAGG & scaffold_29:832388-832402 & $\mathrm{N}$ \\
\hline dpu-miR-3185 & MIMAT0015065_st & AGAAGAAGGCGGUCGGUCUGCGG & scaffold_46:139493-139479 & $\mathrm{N}$ \\
\hline dpu-miR-3201 & MIMAT0015086_st & GGGAUAUGAAGAAAAAU & scaffold_64:179437-179451 & $\mathrm{N}$ \\
\hline dpu-miR-3247 & MIMAT0015429_st & UUAAAUUGUAAAAAAGCGUGGGUU & scaffold_1231:6461-6480 & $\mathrm{N}$ \\
\hline dpu-miR-3269 & MIMAT0015454_st & UGUCUGCAUACAAAAAACAGAA & scaffold_55:380245-380260 & $\mathrm{N}$ \\
\hline dpu-miR-3332 & MIMAT0015519_st & UCCUCGCCAUGCCACCGCCGCUUCA & scaffold_5:1653729-1653743 & $\mathrm{N}$ \\
\hline dpu-miR-3407 & MIMAT0015613_st & UCACUGGGAGAGACGGGAAGUUG & scaffold_72:319594-319609 & $\mathrm{N}$ \\
\hline dpu-miR-3420 & MIMAT0015628_st & UACUGUUAUUGCCUGGCCUCGCUCA & scaffold_110:226257-226274 & $\mathrm{N}$ \\
\hline dpu-miR-3541 & MIMAT0017795_st & UCCCUCCCCCUCACUGCA & scaffold_7:2227093-2227107 & $\mathrm{N}$ \\
\hline dpu-miR-3621 & MIMAT0018002_st & CGCGGGUCGGGGUCUGCAGG & scaffold_249:44994-44981 & $\mathrm{N}$ \\
\hline dpu-miR-3656 & MIMAT0018076_st & GGCGGGUGCGGGGGUGG & scaffold_16:1563383-1563396 & $\mathrm{N}$ \\
\hline dpu-miR-3665 & MIMAT0018087_st & AGCAGGUGCGGGGCGGCG & scaffold_16:1563383-1563396 & $\mathrm{N}$ \\
\hline dpu-miR-3960 & MIMAT0019336_st & GGCGGCGGCGGAGGCGGGGG & scaffold_827:6844-6863 & $\mathrm{N}$ \\
\hline
\end{tabular}




\begin{tabular}{|c|c|c|c|c|}
\hline dpu-miR-4270 & MIMAT0016900_st & UCAGGGAGUCAGGGGAGGGC & scaffold_20:779208-779192 & $\mathrm{N}$ \\
\hline dpu-miR-4296 & MIMAT0016845_st & AUGUGGGCUCAGGCUCA & scaffold_142:172776-172792 & $\mathrm{N}$ \\
\hline dpu-miR-4298 & MIMAT0016852_st & CUGGGACAGGAGGAGGAGGCAG & scaffold_43:544977-544961 & $\mathrm{N}$ \\
\hline dpu-miR-4299 & MIMAT0016851_st & GCUGGUGACAUGAGAGGC & scaffold_115:119886-119872 & $\mathrm{N}$ \\
\hline dpu-miR-4423 & MIMAT0024068_st & AUAGGCACCAAAAAGCAACAA & scaffold_10:1830855-1830841 & $\mathrm{N}$ \\
\hline dpu-miR-4429 & MIMAT0018944_st & AAAAGCUGGGCUGAGAGGCG & scaffold_202:138164-138148 & $\mathrm{N}$ \\
\hline dpu-miR-4430 & MIMAT0018945_st & AGGCUGGAGUGAGCGGAG & scaffold_25:300708-300695 & $\mathrm{N}$ \\
\hline dpu-miR-4440 & MIMAT0018958_st & UGUCGUGGGGCUUGCUGGCUUG & scaffold_3295:3630-3616 & $\mathrm{N}$ \\
\hline dpu-miR-4443 & MIMAT0018961_st & UUGGAGGCGUGGGUUUU & scaffold_59:305314-305327 & $\mathrm{N}$ \\
\hline dpu-miR-4459 & MIMAT0018981_st & CCAGGAGGCGGAGGAGGUGGAG & scaffold_38:797104-797125 & $\mathrm{N}$ \\
\hline dpu-miR-4462 & MIMAT0018986_st & UGACACGGAGGGUGGCUUGGGAA & scaffold_6:700566-700553 & $\mathrm{N}$ \\
\hline dpu-miR-4466 & MIMAT0018993_st & GGGUGCGGGCCGGCGGGG & scaffold_97:160316-160303 & $\mathrm{N}$ \\
\hline dpu-miR-4481 & MIMAT0019015_st & GGAGUGGGCUGGUGGUU & scaffold_21:906810-906797 & $\mathrm{N}$ \\
\hline dpu-miR-4484 & MIMAT0024307_st & GAAAAAGGCGGGAGAAGCCCCA & scaffold_12:977084-977064 & $\mathrm{N}$ \\
\hline dpu-miR-4486 & MIMAT0019020_st & GCUGGGCGAGGCUGGCA & scaffold_135:109548-109535 & $\mathrm{N}$ \\
\hline dpu-miR-4487 & MIMAT0019021_st & AGAGCUGGCUGAAGGGCAG & scaffold_107:395870-395854 & $\mathrm{N}$ \\
\hline dpu-miR-4488 & MIMAT0024152_st & UAGGGGGCGGGCUCCGGCG & scaffold_1:1455216-1455229 & $\mathrm{N}$ \\
\hline dpu-miR-4497 & MIMAT0019032_st & CUCCGGGACGGCUGGGC & scaffold_22:507000-506987 & $\mathrm{N}$ \\
\hline dpu-miR-4507 & MIMAT0019044_st & CUGGGUUGGGCUGGGCUGGG & scaffold_200:122781-122798 & $\mathrm{N}$ \\
\hline dpu-miR-4508 & MIMAT0019045_st & GCGGGGCUGGGCGCGCG & scaffold_106:436230-436216 & $\mathrm{N}$ \\
\hline dpu-miR-4516 & MIMAT0019053_st & GGGAGAAGGGUCGGGGC & scaffold_7:983112-983099 & $\mathrm{N}$ \\
\hline dpu-miR-4530 & MIMAT0019069_st & CCCAGCAGGACGGGAGCG & scaffold_53:169581-169565 & $\mathrm{N}$ \\
\hline dpu-miR-4532 & MIMAT0019071_st & CCCCGGGGAGCCCGGCG & scaffold_8:1091210-1091192 & $\mathrm{N}$ \\
\hline dpu-miR-4577 & MIMAT0019610_st & GGCGUGGAGACCGAAAGCGAACG & scaffold_7:1023185-1023171 & $\mathrm{N}$ \\
\hline dpu-miR-4592 & MIMAT0019625_st & CCAGCGGCGGUGCCGUGAUGGCGA & scaffold_36:135712-135697 & $\mathrm{N}$ \\
\hline
\end{tabular}




\begin{tabular}{|c|c|c|c|c|}
\hline dpu-miR-4606 & MIMAT0019640_st & UGCGUGCGGUGAGGUGGCCGGGG & scaffold_328:49714-49701 & $\mathrm{N}$ \\
\hline dpu-miR-4624 & MIMAT0019658_st & CCGCGGUCGGGUCGGGCUGAGUG & scaffold_114:15246-15232 & $\mathrm{N}$ \\
\hline dpu-miR-4651 & MIMAT0019715_st & CGGGGUGGGUGAGGUCGGGC & scaffold_4691:692-679 & $\mathrm{N}$ \\
\hline dpu-miR-4654 & MIMAT0024053_st & UGUGGGAUCUGGAGGCAUCUGGG & scaffold_43:746811-746826 & $\mathrm{N}$ \\
\hline dpu-miR-4656 & MIMAT0019723_st & UGGGCUGAGGGCAGGAGGCCUGU & scaffold_99:295357-295375 & $\mathrm{N}$ \\
\hline dpu-miR-4689 & MIMAT0019778_st & UUGAGGAGACAUGGUGGGGGCC & scaffold_118:363760-363744 & $\mathrm{N}$ \\
\hline dpu-miR-4706 & MIMAT0019806_st & AGCGGGGAGGAAGUGGGCGCUGCUU & scaffold_245:75030-75047 & $\mathrm{N}$ \\
\hline dpu-miR-4739 & MIMAT0019868_st & AAGGGAGGAGGAGCGGAGGGGCCCU & scaffold_39:594305-594325 & $\mathrm{N}$ \\
\hline dpu-miR-4741 & MIMAT0019871_st & CGGGCUGUCCGGAGGGGUCGGCU & scaffold_3:2595703-2595689 & $\mathrm{N}$ \\
\hline dpu-miR-4743 & MIMAT0024045_st & UGGCCGGAUGGGACAGGAGGCA & scaffold_118:264841-264826 & $\mathrm{N}$ \\
\hline dpu-miR-4847 & MIMAT0020069_st & UAAUGAUGGCGCGGUGCGGUGC & scaffold_307:20602-20589 & $\mathrm{N}$ \\
\hline dpu-miR-4898 & MIMAT0020490_st & GCUGUCCUGCGGCUGGUGGGAGGU & scaffold_177:221915-221931 & $\mathrm{N}$ \\
\hline dpu-miR-4930 & MIMAT0020136_st & GGCUGCCUAGGGGGCUGGCUAG & scaffold_83:219483-219470 & $\mathrm{N}$ \\
\hline dpu-miR-4937 & MIMAT0020143_st & CUGGGUAGAGGUUGGGCGGUGGA & scaffold_60:685657-685674 & $\mathrm{N}$ \\
\hline dpu-miR-5110 & MIMAT0020618_st & GGAGGAGGUAGAGGGUGGUGGAAUU & scaffold_166:173460-173444 & $\mathrm{N}$ \\
\hline dpu-miR-5112 & MIMAT0020620_st & UAGCUCAGCGGGAGAGCAC & scaffold_13611:1015-1033 & $\mathrm{N}$ \\
\hline dpu-miR-5122 & MIMAT0020630_st & CCGCGGGACCCGGGGCUGUG & scaffold_1240:9348-9367 & $\mathrm{N}$ \\
\hline dpu-miR-5126 & MIMAT0020637_st & GCGGGCGGGGCCGGGGGCGGGG & scaffold_1240:9348-9367 & $\mathrm{N}$ \\
\hline dpu-miR-5128 & MIMAT0020639_st & CAAUUGGGGCUGGCGAGAUGGCU & scaffold_15:869554-869568 & $\mathrm{N}$ \\
\hline dpu-miR-5130 & MIMAT0020641_st & CUGGAGCGCGCGGGCGAGGCAGGC & scaffold_52:55617-55635 & $\mathrm{N}$ \\
\hline dpu-miR-5328 & MIMAT0021401_st & UUAGAGGAGUCGAAGCAGCGUUGGCC & scaffold_2:3233149-3233135 & $\mathrm{N}$ \\
\hline dpu-miR-5739 & MIMAT0023116_st & GCGGAGAGAGAAUGGGGAGC & scaffold_76:623190-623175 & $\mathrm{N}$ \\
\hline dpu-miR-5787 & MIMAT0023252_st & GGGCUGGGGCGCGGGGAGGU & scaffold_88:194891-194875 & $\mathrm{N}$ \\
\hline dpu-miR-5952 & MIMAT0023454_st & UGAGGGACUGGUCGCGGCAGAAUA & scaffold_88:513930-513943 & $\mathrm{N}$ \\
\hline dpu-miR-5960 & MIMAT0023464_st & GUGGUCCGGAGUCGGAGGGUUAU & scaffold_158:132518-132502 & $\mathrm{N}$ \\
\hline
\end{tabular}




\begin{tabular}{|l|l|l|l|c|} 
dpu-miR-6048 & MIMAT0023672_st & AGAGGCGAGGUGGACCGACUGGU & scaffold_1391:8726-8740 & $\mathrm{N}$ \\
\hline dpu-miR-6056 & MIMAT0023680_st & GAGGGACGAGGAGGGAGGUU & scaffold_110:246163-246177 & $\mathrm{N}$ \\
\hline dpu-miR-6084 & MIMAT0023709_st & UUCCGCCAGUCGGUGGCCGG & scaffold_377:26518-26533 & $\mathrm{N}$ \\
\hline dpu-miR-6085 & MIMAT0023710_st & AAGGGGCUGGGGGAGCACA & scaffold_20:1377740-1377727 & $\mathrm{N}$ \\
\hline dpu-miR-6086 & MIMAT0023711_st & GGAGGUUGGGAAGGGCAGAG & scaffold_197:125507-125522 & $\mathrm{N}$ \\
\hline dpu-miR-6087 & MIMAT0023712_st & UGAGGCGGGGGGGGGGC & scaffold_16:305737-305754 & $\mathrm{N}$ \\
\hline dpu-miR-6088 & MIMAT0023713_st & AGAGAUGAAGCGGGGGGGGG & scaffold_81:377230-377247 & $\mathrm{N}$ \\
\hline dpu-miR-6089 & MIMAT0023714_st & GGAGGCCGGGGUGGGGCGGGGGG & scaffold_20:380862-380878 & $\mathrm{N}$ \\
\hline dpu-miR-6090 & MIMAT0023715_st & GGGGAGCGAGGGGGGGGC & scaffold_142:277430-277446 & $\mathrm{N}$ \\
\hline dpu-miR-6125 & MIMAT0024598_st & GCGGAAGGCGGAGCGGGGGA & scaffold_4:754467-754484 & $\mathrm{N}$ \\
\hline dpu-miR-6127 & MIMAT0024600_st & UGAGGGAGUGGGUGGGAGG & scaffold_23:587924-587939 & $\mathrm{N}$ \\
\hline
\end{tabular}


Table S5. Predicted miRNA targets.

\begin{tabular}{|c|c|c|}
\hline miRNA Name & Predicted Target gene & Gene Symbo \\
\hline \multirow{9}{*}{ miR-210 } & Calcium-transporting ATPase type $2 \mathrm{C}$ member & ATP2C2 \\
\hline & Hypoxia up-regulated protein 1 & HYOU1 \\
\hline & Small conductance calcium-activated potassium channel protein & KCNN2 \\
\hline & Potassium/sodium hyperpolarization-activated cyclic nucleotide-gated channel 2 & HCN2 \\
\hline & Copper-transporting ATPase 1 & ATP7A \\
\hline & Sodium channel protein type 2 subunit alpha & SCN2A \\
\hline & Mucin and cadherin-like protein & MUPCDH \\
\hline & Glyceraldehyde-3-phosphate dehydrogenase & GAPDH \\
\hline & DNA mismatch repair protein Mlh3 & MLH3 \\
\hline \multirow{9}{*}{$\operatorname{miR}-71$} & Calcium-transporting ATPase sarcoplasmic/endoplasmic reticulum type & Ca-P60A \\
\hline & High affinity copper transporter & SLC31A1 \\
\hline & Sodium channel protein type 2 subunit alpha & SCN2A \\
\hline & Phosphatidylinositol-4-phosphate type-2 alpha & $P I 4 K 2 A$ \\
\hline & C-type lectin & CLEC \\
\hline & GLIS family zinc finger 1 & GLIS1 \\
\hline & Transcription factor hes-1 & HES1 \\
\hline & ATP-binding cassette sub-family D member 2 & $A B C D 2$ \\
\hline & ATP-binding cassette sub-family A member 3 & $A B C A 3$ \\
\hline \multirow{6}{*}{ miR-252 } & ATP-binding cassette sub-family G member 1 & $A B C G 1$ \\
\hline & Mitochondrial ribosomal protein s31 & MRPS31 \\
\hline & Phosphatidylinositol-4-phosphate type-2 alpha & PI4K2A \\
\hline & Heat shock transcription factor 1 & HSF1 \\
\hline & Histone h4 replacement & HIS4R \\
\hline & Mitochondrial ribosomal protein L10 & MRPL10 \\
\hline \multirow{6}{*}{$\operatorname{miR}-279$} & ATP-binding cassette sub-family G member 1 & $A B C G 1$ \\
\hline & Calcium-transporting ATPase sarcoplasmic/endoplasmic reticulum type & Ca-P60A \\
\hline & Myosin IXa protein & MYO9A \\
\hline & Myosin-2 heavy chain & $\mathrm{MYH2}$ \\
\hline & Myosin light chain kinase & MLCK \\
\hline & DNA repair and recombination protein RAD54 & RAD54 \\
\hline
\end{tabular}

Table S6. Gene symbols used in IPA network analysis attributed to the Homo sapiens annotations. Letters denote if these genes have homologs in $D$. pulex.

\begin{tabular}{|l|l|l|}
\hline Symbol & Entrez Gene Name & $\begin{array}{l}\text { D. pulex } \\
\text { homolog (Y/N) }\end{array}$ \\
\hline ADAM10 & ADAM metallopeptidase domain 10 & Y \\
\hline BBC3 & BCL2 binding component 3 & $\mathrm{N}$ \\
\hline CELF2 & CUGBP, Elav-like family member 2 & $\mathrm{Y}$ \\
\hline
\end{tabular}




\begin{tabular}{|l|l|l|} 
DACH1 & dachshund family transcription factor 1 & Y \\
\hline DDX20 & DEAD (Asp-Glu-Ala-Asp) box polypeptide 20 & Y \\
\hline DICER1 & dicer 1, ribonuclease type III & Y \\
\hline EIF2AK3 & eukaryotic translation initiation factor 2-alpha kinase 3 & Y \\
\hline ELK3 & ELK3, ETS-domain protein (SRF accessory protein 2) & Y \\
\hline EPHA3 & EPH receptor A3 & Y \\
\hline FOXC1 & forkhead box C1 & Y \\
\hline HDL & high-density lipoprotein & Y \\
\hline HIPK3 & homeodomain interacting protein kinase 3 & Y \\
\hline HNF1B & HNF1 homeobox B & Y \\
\hline IgG2b & immunoglobulin heavy constant gamma 2B & $\mathrm{N}$ \\
\hline LTBP1 & latent transforming growth factor beta binding protein 1 & $\mathrm{Y}$ \\
\hline MAP2K4 & mitogen-activated protein kinase kinase 4 & $\mathrm{Y}$ \\
\hline Nfat (family) & Nuclear factor of activated Tcells & \\
\hline & $\begin{array}{l}\text { prostaglandin-endoperoxide synthase 2 (prostaglandin G/H } \\
\text { synthase and cyclooxygenase) }\end{array}$ & $\mathrm{Y}$ \\
\hline PTGS2 & regulator of G-protein signaling 5 & $\mathrm{Y}$ \\
\hline RGS5 & $\begin{array}{l}\text { solute carrier family 7 (amino acid transporter light chain, L } \\
\text { system), member 5 }\end{array}$ & $\mathrm{Y}$ \\
\hline & transforming growth factor, beta 1 & $\mathrm{Y}$ \\
\hline SLC7A5 & thioredoxin interacting protein & $\mathrm{Y}$ \\
\hline TGFB1 & zinc finger E-box binding homeobox 2 & $\mathrm{Y}$ \\
\hline TXNIP & & \\
\hline ZEB2 & & \\
\hline
\end{tabular}

